\title{
A NEW CASE OF DIPLOIDY WITHIN A HAPLOID GENUS OF ENTOMOPATHOGENIC FUNGI
}

Authors and affiliations:

Knud Nor Nielsen ${ }^{1 *}$, João Felipe Moreira Salgado ${ }^{2,3}$, Myrsini Eirini Natsopoulou ${ }^{1}$, Jason E Stajich ${ }^{2}$, Henrik H. De Fine Licht ${ }^{1}$

Section for Organismal Biology, Department of Plant and Environmental Sciences, University of Copenhagen, 1871 Frederiksberg C, Denmark

Department of Microbiology and Plant Pathology, University of California Riverside, Riverside, CA, 92521 USA

Instituto de Microbiologia Paulo de Góes, Universidade Federal do Rio de Janeiro, Rio de Janeiro, Brazil

Corresponding author: knn@plen.ku.dk 


\begin{abstract}
Fungi in the genus Metarhizium are soil-borne plant-root endophytes and rhizosphere colonisers, but also potent insect pathogens with highly variable host ranges. These ascomycete fungi are predominantly asexually reproducing and ancestrally haploid, but two independent origins of persistent diploidy within the Coleoptera-infecting M. majus species complex are known and has been attributed to incomplete chromosomal segregation following meiosis during the sexual cycle. There is also evidence for infrequent sexual cycles in the locust-specific pathogenic fungus Metarhizium acridum (Hypocreales: Clavicipitaceae), which is an important entomopathogenic biocontrol agent used for the control of grasshoppers in agricultural systems as an alternative to chemical control. Here, we show that the genome of the M. acridum isolate ARSEF 324, which is formulated and commercially utilised under the name 'Green Guard', is functionally diploid. We used single-molecule real-time (SMRT) sequencing technology to complete a high-quality assembly of ARSEF 324. Kmer frequencies, intragenomic collinearity between contigs and single nucleotide variant read depths across the genome revealed the first incidence of diploidy described within the species $M$. acridum. The haploid assembly of $44.7 \mathrm{Mb}$ consisting of $20.8 \%$ repetitive elements, which is the highest proportion described of any Metarhizium species. The genome assembly and the inferred diploid state, can shed light on past research on this strain and could fuel future investigation into the fitness landscape of aberrant ploidy levels, not least in the contest of biocontrol agents.
\end{abstract}

Key words: Genome duplication, Metarhizium, fungus, ascomycete

\title{
INTRODUCTION
}

The duplication of single genes, genomic segments, chromosomes, and whole-genome duplications (WGD) has played a vital role in eukaryotic evolution by providing genetic material for adaptation. Throughout the evolution, recurrent WGD events have been linked to the emergence, diversification and survival of species (Crow and Wagner, 2006). For example, WGD has been coupled with the emergence of angiosperms within plants (De Bodt, Maere and Van de Peer, 2005), and with the emergence of vertebrates, gnathostomes and teleosts within metazoan chordate evolution (Sacerdot et al., 2018).

A well-described ancient WGD event in the fungal kingdom is the ancient diploidisation that lead to the formation of the Saccharomyces genus (Wolfe and Shields, 1997; Dietrich, 2004; Kellis, Birren and Lander, 2004). While some WGD events lead to stable polyploids, as in the case of Saccharomyces where an ancient hybridization event is thought to have provided stability and fertility to the new diploid (Marcet-houben and Gabaldón, 2015), duplications are usually perceived as transient. Duplicated genes enter into a cycle with subsequent reductions by loss or mutational decay leading to pseudogenisation or neofunctionalisation (Lynch, 2000; Levasseur and Pontarotti, 2011). It should be noted that it can be difficult to distinguish ancient WGD events from cases of multiple segmental duplications caused by transposon activity (Roelofs et al., 2020). Regardless of origin, duplications have long been recognised as a driver of evolution by providing the material for natural selection to work on (Bridges, 1919; Metz, 1947; Ohno, 1970; Crow and Wagner, 2006).

WGD arise from abnormal cell cycles post chromosome duplication, either by the absence of mitotic division or cytokinesis. The duplication itself can occur by auto- or allopolyploidisation. The latter 
involves the fusion of individual cells and subsequent fusion of nuclei bringing together the variants accumulated between the genetically distinct individuals. In contrast, autopolyploidisation, or endoreplication, only involves a single cell and has been associated with environmental stress. Within plants, several studies have shown that endoreplication can be induced by abiotic stress, such as heat (Monjardino, Smith and Jones, 2006), drought (Cookson, Radziejwoski and Granier, 2006), elevated salinity (Barkla et al., 2018), and toxins (Biskup and Izmaiłow, 2004). The duplication is thought to mediate an acute increase in the transcription of metabolic and stress-mitigating genes (Scholes and Paige, 2015). The human pathogenic fungi Candida albicans and Cryptococcus neoformans have been shown to repeatedly gain resistance to antifungal drugs such as fluconazole through chromosomal duplications (Sionov et al., 2010; Kronstad et al., 2011). C. neoformans can also evade phagocytosis within the lungs by creating titan cells (Okagaki et al., 2010; Zaragoza et al., 2010). The increased cell size is induced by multiple rounds of endoreplication and is observed in up to one-fifth of the $C$. neoformans cells residing in infected lung tissue (Okagaki et al., 2010). Transient somatic endoreduplication has also been recognised as a driver of developmental change of some cell types in plants, insects and mammals (Fox and Duronio, 2013). Examples of somatic endoreplication include the giant cells of the mammalian trophoblast that develops into a significant part of the placenta, and the $>1000 x$ sister chromatids observed in the salivary glands of Drosophila melanogaster (Sher et al., 2013).

Within fungi, increasing evidence suggests widespread cryptic population-level ploidy variations. Whole-genome sequencing of an extensive collection of 794 natural (wild) isolates of Saccharomyces cerevisiae found alternative ploidy levels within $13 \%$, of the otherwise predominately diploid yeast (Peter et al., 2018). The same study found chromosomal copy number variation (CCNV or aneuploidy) in 19\% of the isolates (193 of the 1,011), reiterating the importance of variation in chromosome numbers for organismal evolution (Mayrose and Lysak, 2020). Although at least some fungi readily undergo endoreplication such levels of genome plasticity is not without constraints. A long-term evolutionary experiment with $S$. cerevisiae showed a convergence from alternative ploidies to the ancestral diploid form (Gerstein et al., 2006). The chytrid fungus Batrachochytrium dendrobatidis threatening amphibians worldwide has a high rate of CCNV in nature (Rosenblum et al., 2013). This plasticity has been linked to pathogenicity through lab experiments which showed a gain of chromosomal copies upon induced stress by a characteristic host antimicrobial peptide (Farrer et al., 2013).

While the majority of known fungi are dikaryotic at some stage, diploidy is the exception. Filamentous ascomycetes (Pezizomycotina) are only dikaryotic in specific life stages, namely the ascogenous hyphae and ascocarp, while the bulk of the mycelium remains monokaryotic, and in the majority of species, haploid. Exceptions to haploidy within this important group of fungi are found in the Phyllactinia, Stephensia, Xylaria, Botrytis and Zygosaccharomyces genera (Albertin and Marullo, 2012), and within the genus Metarhizium (Kepler et al., 2016). When these fungi contain two different idiomorphs of the mating-type genes within the same diploid, it indicates mating events that failed to complete meiosis, leading to the observed allopolyploidisation. Diploidy may also arise from the parasexual cycle of fungi, which allows for nuclei fusion (karyogamy) following anastomosis of strains from somatically compatible groups. Contrary to the sexual cycle, fused nuclei of the parasexual cycle do not undergo meiosis, but continue to divide mitotically. The original ploidy level is restored by random chromosome loss, through a series of aneuploidy intermediates (Moore, Robson and Trinci, 2011), which is known from the 'asexual' human pathogen Candida albicans. Reversion is a gradual 
process with generations of aneuploidy. Sexual recombination requires different mating-types, whereas the parasexual cycle normally requires compatible identities. The reproductive isolation of the parasexual cycle is enforced by heterokaryon incompatibility protein (HET) domains that abort incompatible hyphal fusion attempts (Glass and Dementhon, 2006). This entails that any parasexual fusion of nuclei will be of closely related and highly similar genomes. Infected insects can be hot spots of fungal parasexuality, and the formation of diploid conidia (Riba et al., 1980; Leal-Bertioli et al., 2000; Wang et al., 2011). Investigating the conidida harvested from an insect coinfected with two fluorescently labelled isolates of the same $M$. robertsii strain revealed that $24 \%$ of the conidia investigated were diploid (Wang et al., 2011). Similar observations have been reported from experiments with M. anisopliae and M. majus (Riba et al., 1980; Leal-Bertioli et al., 2000).

The entomopathogenic fungus M. acridum (Hypocreales: Clavicipitaceae) is a specialist with a narrow host range of insects of the order Orthoptera. Host specificity is enforced by the requirement of Orthopteran cuticle signals facilitating spore germination and appressorial formation (Wang and St. Leger, 2005). The M. acridum strain ARSEF 324 is the active ingredient in the biocontrol agent 'Green Guard' targeting grasshoppers (Acrididae), which is commercially deployed in Africa, Australia and China where these insects are agricultural pests of concern. Within the genus Metarhizium, $M$. acridum, as well as the specialist M. album, are considered to be cryptic sexual species (Hu et al., 2014; St. Leger and Wang, 2020). No teleomorphs are known, but genomic analyses have found signatures of an active repeat-induced point mutations (RIP) machinery, an indirect sign of meiosis and sexual recombination (Galagan and Selker, 2004). This is in contrast to the co-generic generalists with wide host ranges, $M$. pingshaense, $M$. anisopliae, $M$. robertsii and $M$. brunneum (the PARB clade, sensu Bischoff et al. 2009), where no RIP footprint is present, consequently causing these species to be considered primarily asexual.

The ascomycete genus Metarhizium is generally haploid, but Kepler et al. (2014) found two independent origins of stable diploid clades interspersed among lineages comprised entirely of haploid individuals within the larger Metarhizium guizhouense/majus/taii clade (MGT clade; sensu Bischoff et al., 2009). All isolates within these diploid taxa were consistently heterozygotic for the analysed microsatellite markers and contained two different ideomorphs of the mating-type genes (MAT1 and MAT2). The presence of both mating-types was interpreted as a mating event that failed to complete meiosis, which lead to the observed allopolyploidisation. The locust-specific $M$. acridum has so far been regarded as entirely haploid, and the only previously available genome for this species was from the haploid strain CQMa 102. This strain was sequenced and assembled into 241 scaffolds (>1 kb; N50, $329.5 \mathrm{~kb}$ ) containing 1,609 contigs with a total genome size of $38.0 \mathrm{Mb}$ (Gao et al., 2011).

In this study, we describe another instance of diploidy within the genus Metarhizium and the first within M. acridum. We present a de-duplicated haploid genome assembly of $M$. acridum strain ARSEF 324 , which consist of 35 contigs representing a haploid genome of $44.7 \mathrm{Mb}$. Ploidy is established through $\mathrm{K}$-mer analysis on unassembled sequencing reads, and intragenomic collinearity analysis by all-vs-all mapping of contigs. Euploidy was established through sequencing read depth comparison across each contig, and through estimation of heterozygosity ratios of single nucleotide variants (SNV), both in $10 \mathrm{~Kb}$ non-overlapping sliding windows. Finally, using DAPI fluorescence staining we demonstrate that conidia are uninucleate. 


\section{MATERIALS AND METHODS}

\section{Strain and culturing}

The Metarhizium acridum strain ARSEF 324 was isolated in 1979 from a spur-throated locust (Austracris guttulosa) in Australia and obtained from the ARSEF collection (ARS Collection of Entomopathogenic Fungal Cultures, Ithaca, New York). A single-spore isolate was obtained by plating serial-dilutions of conidia from the original culture. Conidia from the single-spore culture were grown in liquid sabouraud dextrose broth with yeast extract $(40 \mathrm{~g} / \mathrm{L}$ dextrose, $10 \mathrm{~g} / \mathrm{L}$ peptone, $10 \mathrm{~g} / \mathrm{L}$ yeast extract, $\mathrm{pH}=6.5$ ) while being stirred at $170 \mathrm{rpm}$ on a shaking table for 24 hours before DNA extraction. Fluorescence staining with DAPI (blue, 4',6-diamidino-2-phenylindole) was used to visualise the number of nuclei within mature conidia, as described in (Kepler et al., 2016). Imaging was done on a Zeiss Axioscope microscope (x100 objective) with an AxioCam ICm1, and spores measured using ImageJ v1.53e (Schneider, Rasband and Eliceiri, 2012).

\section{DNA and RNA extraction and sequencing}

Liquid culture filtrate was ground with liquid nitrogen in a mortar and ca. $500 \mathrm{mg}$ finely ground powder transferred to $50 \mathrm{~mL}$ Falcon tube. DNA was extracted using a CTAB method by adding $17.5 \mathrm{~mL}$ CTAB buffer with $0.1 \%$ 2-Mercaptoethanol and $125 \mu \mathrm{l}$ Proteinase $\mathrm{K}$ and incubated for one hour at $60{ }^{\circ} \mathrm{C}$. After centrifugation for $20 \mathrm{~min}$. at $5.000 \mathrm{~g}$ at $4{ }^{\circ} \mathrm{C}$, the supernatant was removed and washed with 1 volume of Phenol/chloroform/isoamyl alcohol (25:24:1) followed by two rounds of 1 volume chloroform/isoamyl alcohol (24:1) and centrifuged for $10 \mathrm{~min}$ at $11.000 \mathrm{rpm}$ at each step. To remove RNA, $150 \mu$ l RNAse was added to the aqueous phase and incubated for $120 \mathrm{~min}$. at $37^{\circ} \mathrm{C}$. To precipitate DNA, 0.6 vol. 2-propanol was added and incubated at $-20^{\circ} \mathrm{C}$ overnight, before centrifugation at 11.000 $\mathrm{rpm}$ at $4{ }^{\circ} \mathrm{C}$ for $30 \mathrm{~min}$. The resulting pellet was washed twice with $2 \mathrm{~mL} 70 \%$ Ethanol and centrifuged for $10 \mathrm{~min}$ at $11.000 \mathrm{rpm}$ at $4{ }^{\circ} \mathrm{C}$ before being suspended in $500 \mu \mathrm{l} \mathrm{TE}$-buffer. Purity was checked using NanoDrop reading and DNA quantity using a Qubit Broad-Range analysis kit.

A single SMART Cell library was sequenced on a PacBio Sequel platform using the Sequel ${ }^{\circledR}$ Sequencing Kit 2.1 v2 (Sequencing Kit par number 101-309-500). Each continuous long read (CLR) had one passage of sequencing; subsequently, subreads were generated by the removal of adapters and bases were called with the basecaller v5.0.0.6236. Sequencing was performed by Genewiz (Takeley, UK) with a yield from the single SMRT Cell equaling a coverage of $134 \mathrm{x}$, assuming a $45 \mathrm{Mb}$ genome, with a mean subread length of $11.2 \mathrm{~Kb}$. Short read sequence data was obtained using the PCR-free DBNseq ${ }^{\mathrm{TM}}$ platform at BGI-tech Copenhagen. Sequencing adapters were removed by the sequencing company, delivering a total of $15,111,206$ reads of $150 \mathrm{bp}$., equaling a coverage of $50 \mathrm{x}$, assuming a $45 \mathrm{Mb}$ genome.

To obtain RNAseq samples to aid in the genome prediction, four replicate flasks with $20 \mathrm{~mL}$ media were grown for four days as described above but with $100 \mathrm{rpm}$ agitation. After filtration through a Whatman filter paper (5-13 $\mu \mathrm{m})$, the fungal material was collected and flash-frozen in liquid nitrogen before being pulverised in a tissue lyzer and extracted using a QIAGEN plant RNeasy kit following manufacturers specifications. The four samples were sequenced separately using the DBNseq ${ }^{\mathrm{TM}}$ platform at BGI-tech Copenhagen yielding ca. 16,000,000 paired-end 150 bp reads per sample. 


\section{K-mer analysis and genome assembly}

K-mers frequencies within PacBio subread data was established with KMC 3 (Kokot, Długosz and Deorowicz, 2017), and genome size and ploidy were inferred using GenomeScope v2.0 (RanalloBenavidez, Jaron and Schatz, 2020). Ploidy was further investigated comparing the sum of kmer pair coverages using Smudgeplot implemented in GenomeScope. The mitochondrion was assembled from 2806 reads mapping to the mitochondrion of Metarhizium rileyi strain RCEF 4871 (NCBI assession: NC_047289.1; Zhang et al., 2020). The alignment was done with Minimap2 v2.17(r941) (Li, 2018). Only reads above eight $\mathrm{kb}$, which mapped with more than $70 \%$ of their length was kept. The mitochondrion was assembled using Canu v. 2.0 (Koren et al., 2017) with the settings: 'genomeSize $=62 k^{\prime}$ 'corOutCoverage=999'.

Sequence reads were assembled with Canu v. 2.0, using the following parameters to co-assemble haplotypes: genomeSize $=45 \mathrm{~m}$ correctedErrorRate $=0.03$ corOutCoverage $=200$ batOptions $=-\mathrm{dg} 3-\mathrm{db} 3$ -dr 1 -ca 500 -cp 50. Two rounds of polishing were conducted to improve the assembly, each consisting of mapping raw-reads to the assembly using Minimap2 v.2.6 (Li, 2018), and reducing remaining insertion-deletion and base substitution errors by polishing the consensus sequence using Arrow v2.3.3 (https://github.com/PacificBiosciences/GenomicConsensus). Mirror-reads (putatively an effect of missing adapters during sequencing) were detected in the PacBio subreads set; therefore, reads longer than $40 \mathrm{~kb}$ were filtered out prior to assembly. Different levels of the correctedErrorRate parameter were tested (i.e.: $0.020,0.025,0.030,0.035,0.040$ ). Each of the five assemblies was assessed based on the complementarity of phased contigs. The value 0.030 were chosen for the final assembly (see sup. figure 1).

Contigs were ordered according to length and grouped into primary contigs, forming a haploid representative genome assembly (Haplotig $1(\mathrm{H} 1)$ ) and shorter contigs $(\mathrm{H} 2)$ that mapped to the primary contigs. Primary contigs were identified by an all-versus-all contig mapping on the repeat masked assembly with minimap2 v.2.17. The best match of each contig to a longer contig was assessed from the accumulated length of alignment between each pair of contigs. Primary contigs were manually curated; three contigs were not only subjects to mapping, but also query to other primary contigs. These contigs, and the regions they mapped to had approximately half the read depth of the mean read depth of the other primary contigs. Since the read depth of the subject regions could be raised to the mean read depth, if the query contigs were removed before mapping, these three contigs were assumed to be haplotigs and moved to the $\mathrm{H} 2$ group.

\section{Transcriptome assembly}

Adapter and low quality sequences were trimmed from RNA-seq reads with Trimmomatic v.0.36 (Bolger, Lohse and Usadel, 2014) and poly-A sequences were clipped from transcripts using SeqClean [available at http://compbio.dfci.harvard.edu/tgi/software/]. Reads were aligned to the complete assembled genome ( $\mathrm{H} 1+\mathrm{H} 2)$ of M. acridum ARSEF 324 using HISAT2 (Kim et al., 2019), allowing for a maximum intron length of $3 \mathrm{~kb}$. This was followed by clustering of aligned reads using Trinity v2.8.5 (Grabherr et al., 2011) in a genome-guided de novo transcriptomic assembly, using the jaccard clip parameter to reduce transcript fusion. The Trinity assembled transcripts were input to gene prediction training to support genome annotation. 


\section{Genome annotation}

A custom repeat library was created by adding de novo identified repeats from RepeatModeler (Hubley et al., 2016) to the repeat databases, Dfam_Consensus-20170127 (Hubley et al., 2016) and RepBase-20170127 (Bao, Kojima and Kohany, 2015). RepeatModeler was run on the genome masked by these two public databases, and the iteratively growing custom library, using RepeatMasker v4.0.7 (Smit, Hubley and Green, 2015) with the option [-species] set to fungi. RepeatModeler relies on the three de novo repeat finding programs RECON (Bao and Eddy, 2002), RepeatScout (Price, Jones and Pevzner, 2005) and LtrHarvester/Ltr_retriever (Ellinghaus, Kurtz and Willhoeft, 2008). Repeats were identified using RepeatMasker [option: sensitive] and the generated custom repeat library. The custom library was built on the following four Metarhizium strains: M. acridum CQMa 102, $M$. anisopliae JEF-290, M. brunneum ARSEF 4556, M. rileyi ARSEF 4871.

Gene prediction and functional annotation of the polished assembly was conducted using the Funannotate pipeline v1.8.4 (https://github.com/nextgenusfs/funannotate). Repeats were identified with RepeatModeler and soft masked using RepeatMasker. Protein evidence from a UniProtKB/SwissProt-curated database (v2021_01) (Bateman et al., 2021) was aligned to the genomes using tBLASTn and Exonerate (Slater and Birney, 2005). Two gene prediction tools were used: AUGUSTUS v3.3.3 (Stanke and Morgenstern, 2005) and GeneMark-ES v4.33 (Besemer and Borodovsky, 2005), with Fusarium graminearum as the model for the AUGUSTUS gene predicter and BAKER1 (Hoff et al., 2016) for the training of GeneMark-ES. tRNAs were predicted with tRNAscan-SE (Lowe and Eddy, 1997). Consensus gene models were found with EvidenceModeler (Haas et al., 2008). Gene models were further updated with the 'update' step in funannotate which uses the tool PASA (Haas et al., 2008) to further extend untranslated regions and identify alternatively spliced isoforms based on alignments of RNA-Seq assembled transcripts. The completeness of the assembled genome was evaluated through comparison to the 4494 single-copy ortholog genes of the hypocreales_odb10 dataset (Creation date: 2020-08-05, https://busco-data.ezlab.org/v5/data/lineages/), using BUSCO v5 (Simão et al., 2015).

Functional annotations for the predicted proteins were obtained using BLASTP to search the UniProt/SwissProt protein database (v2021_01). Protein families (Pfam) and Gene Ontology (GO) terms were assigned with InterProScan v5.48-83.0 (Jones et al., 2014). Functional predictions were also inferred by alignments to the eggNOG 5 orthology database (Huerta-Cepas et al., 2019) using emapper v2.0.1b-2-g816e190(Huerta-Cepas et al., 2017). The secretome was predicted using SignalP v5.0 (Almagro Armenteros et al., 2019) and Phobius v1.01 (Käll, Krogh and Sonnhammer, 2007) identifying proteins carrying a signal peptide. To further characterise the secretome, putative CAZymes were identified using HMMER v3.3 (Eddy, 2011) and family-specific hidden Markov model (HMM) profiles of dbCAN2 meta server (Lombard et al., 2014). Putative proteases and protease inhibitors were predicted using the MEROPS database (2017-10-04) (Rawlings, Barrett and Finn, 2016). Biosynthetic Gene Clusters (BGCs) were annotated using strict parameters of the antibiotics and Secondary Metabolites Analysis Shell v5 (antiSMASH) (Blin et al., 2019). All functional predictions and annotations were executed through the Funannotate pipeline.

The guanine-cytosine (GC) content was determined in non-overlapping windows across the $35 \mathrm{H} 1$ contigs, using seqkit v0.13.2 (seqkit sliding -s 100 -W 100 | seqkit fx2tab --name -gc). Each window was annotated with the gene or repeat annotation (or no annotation) which it primarily overlapped. This was achieved with bedtools v.2.28.0 option [intersect -wa - wb - $\mathrm{f} 0.51$ ]. 


\section{Variant calling and chromosome copy number variation (CCNV)}

The 150 bp PE DBNseq reads were mapped with the Burrow-Wheeler Aligner, BWA-MEM v.0.7.16a $(\mathrm{Li}, 2013)$ to the primary haplotig $(\mathrm{H} 1)$ of the assembled nuclear genome, and PCR duplicates were removed from the bam file using samtools v1.10 (markdup -r) (Li et al., 2009). Coverage and mean depth to reference was assessed using samtools (coverage). Allele frequency distribution across the genome was calculated based on single nucleotide variants called using Bcftools v1.10.2 (Li, 2011a). Variants were called by using a combination of BCFtools' mpileup' and 'call' using a mapping quality filter of 30 , a base quality filter of 20 , and a minimum depth of 10 , together with default parameters including BAQ (Li, 2011b). Mean SNV allele ratios were summarised in non-overlapping $10 \mathrm{~kb}$ windows using a custom R script. Genome annotations and repeat annotations was combined for the matingtype loci including the flanking APN2 and SLA2 genes, for to the genome assemblies of $M$. acridum ARSEF 324 and M. acridum CQMa 102 (GenBank accession no. GCA_000187405.1). Synteny between loci was visualised using the R package genoPlotR v0.8.10 (Guy, Roat Kultima and Andersson, 2010).

\section{Genome comparison}

Single copy orthologous genes were identified between $\mathrm{H} 1$ and $\mathrm{H} 2$, and 11 Metarhizium isolates representing eight species, along with two isolates of Pochonia chlamydosporia and two isolates used as outgroup, i.e: Epichloe festucae strain Fl1, and Villosiclava virens strain UV-8b. This was done using OrthoFinder V2.2.7 (Emms and Kelly, 2019). The proteomes of the 16 isolates was obtained from NCBI GenBank; accession numbers are available in the supplementary table 2. Protein sequence alignment was generated with MAFFT v 7.453 as implemented in OrthoFinder. Substitution models for each gene were predicted using ModelFinder (Kalyaanamoorthy et al., 2017) as implemented in IQtree v2.1.2. A subset of 444 known genes with less than $10 \%$ gaps within the alignment was concatenated and a maximum likelihood phylogeny with gene specific substitution models using IQtree (Nguyen et al., 2015; Chernomor, von Haeseler and Minh, 2016).

Repeat-Induced point (RIP) mutations indices were calculated using 'The RIPper' (http://theripper.hawk.rocks). Three different indices were calculated, based on dinucleotide frequencies: RIP substrate [ $(\mathrm{CpA}+\mathrm{TpG}) /(\mathrm{ApC}+\mathrm{GpT})]$ ]: $0.75 \geq \mathrm{x}$, RIP product [ TpA / ApT ]: $x \geq 1.1$ and RIP composite [ (TpA/ApT)-((CpA $+\mathrm{TpG}) /(\mathrm{ApC}+\mathrm{GpT}))]: \mathrm{x} \geq 0$, where $x$ are values indicating RIP on a given sequence. Conservative estimates the RIP affected proportion of the genome are given as regions where all of the above indices indicate RIP (Figure 5).

\section{RESULTS}

\section{K-MER analysis}

The k-mer analysis on the PacBio subreads predicted that the sequenced genome was diploid, and with a haploid length of $45 \mathrm{Mb}$ (Figure $1 \mathrm{~A}$ ). The profile in figure $1 \mathrm{~A}$ shows two peaks in the frequency of observed unique 21-mers within the sequencing data. The smaller peak, has half the read coverage of the taller peak indicating the presence of heterozygous loci. The genome is estimated to have a heterozygosity rate of $0.5 \%$. Analysing the sum of kmer pair coverages to coverage ratios confirmed the inferred diploidy of the data figure 1B (For full GenomeScope results see supplementary material table 1). 
bioRxiv preprint doi: $\mathrm{https}$ ://doi.org/10.1101/2021.03.15.435421; this version posted March 16, 2021. The copyright holder for this preprint (which was not certified by peer review) is the author/funder, who has granted bioRxiv a license to display the preprint in perpetuity. It is made available under aCC-BY-NC-ND 4.0 International license.

\section{Genome assembly}

The mitochondrion genome was assembled to a length of $96,363 \mathrm{bp}$ and contained 17 genes. The number and order of genes corresponds with the conserved structure of mito-genomes within Hypocreales (Aguileta et al., 2014). A total of 478,156 filtered Pacbio reads with an average length of $12.7 \mathrm{~kb}$ was used to assemble the genome into 35 primary contigs representing a haploid genome of $44.71 \mathrm{Mb}$. The primary assembly $\mathrm{H} 1$ has an $\mathrm{L} 50$ of 6 , with a $\mathrm{N} 50$ of $23.37 \mathrm{Mb}$, and 4276 complete BUSCO genes, out of the 4494 BUSCO genes sought, equal to $95.1 \%$ (Scores in BUSCO format: $\mathrm{C}: 95.1 \%[\mathrm{~S}: 94.2 \%, \mathrm{D}: 0.9 \%], \mathrm{F}: 1.4 \%, \mathrm{M}: 3.5 \%, \mathrm{n}: 4494)$. Out of the $44.7 \mathrm{Mb}$ in $\mathrm{H} 1,9.3 \mathrm{Mb}$ (20.8\%) was repetitive elements $74.2 \%$ of which with a strong GC-bias $(<33.2 \%)$.

The alternative haplotig $(\mathrm{H} 2)$ is represented by 565 contigs with a collective size of $39.24 \mathrm{Mb}$, i.e $88 \%$ of the assumed haploid length. This assembly has an L50 of 144, with a N50 of $0.08 \mathrm{Mb}$. This set of contigs only contains loci sufficiently divergent from $\mathrm{H} 1$ to be forked out during the genome assembly. This implies that conserved loci between $\mathrm{H} 1$ and $\mathrm{H} 2$ will be present in the $\mathrm{H} 1$ haplotigs, but largely absent from the $\mathrm{H} 2$. This is reflected in the associated BUSCO scores where $43.7 \%$ of the BUSCO genes are missing from $\mathrm{H} 2$ ( $\mathrm{C}: 54.1 \%[\mathrm{~S}: 51.7 \%, \mathrm{D}: 2.4 \%], \mathrm{F}: 2.2 \%, \mathrm{M}: 43.7 \%, \mathrm{n}: 4494)$. The outer band of the outer tracks of figure 2 depict the size of the 35 primary contigs representing the complete haploid genome. The second band depicts the mapping of the $565 \mathrm{H} 2$ contigs to $\mathrm{H} 1$, indicating their redundancy (Figure 2).

Ideally, the sequencing of an euploid genome should result in an equal read depth across the genome. We mapped both long and short-read data to the $\mathrm{H} 1$ assembly, to assess CCNV. The genome wide mean read depth was $54 x$ and $103 x$ (excluding $>500 x$ depth, at repetitive elements), for long and short read data respectively (Figure 2$)$. Smaller contigs $(<400 \mathrm{~kb}$ ) tended to have lower read depth, likely as a result poor mapping in repeat dense regions. Contig 34 deviates from the general picture with a mean long-read depth of 52x, the lowest mean depth observed. Contig 34 has a $\mathrm{H} 2$ contig that uniquely maps to it, indicating that it is unlikely that contig 34 is a haploid small chromosome. It is possible that it constitutes a haploid segment in the otherwise diploid chromosome. The equal depth across the genome is a strong indication of euploidy across all chromosomes represented in the assembled contigs.

The ploidy of each contig was also assessed by mean ratios of SNV read depth in $10 \mathrm{~kb}$ non-overlapping windows. The expectation is that chromosomes with an even ploidy will tend towards a 50:50 distribution across each SNV, while chromosomes with odd ploidy will tend towards a 33:66 or 33:33:33 ratio. All contigs have distribution of ratios with means of 0.5 (50:50) (Figure 2), confirming the diploidy of all contigs. Deviations from 0.5 correlate with read depth of mapped short-read DBNseq data on which the SNV data is based. Mapping of short-reads likewise correlates with the repeat density (Figure 2), because high repeat densities impede the mapping.

The mean GC content across the $44.7 \mathrm{Mb}$ of the $\mathrm{H} 1$ contigs is $45 \%$ (figure 2, track 5). The GC-content analysed in $100 \mathrm{bp}$ windows shows a bi-modal distribution with peaks at $17.6 \%$ and $51.3 \%$ (Figure $3 \mathrm{~A}$ ). In the following we delimitate the low and high GC peaks at $33.2 \%$. Repetitive elements explain $81.5 \%$ of the low GC peak, $40.5 \%$ are unknown repetitive elements, simple repeats $28.7 \%$, retroelements $6.4 \%$, and DNA transposons explain $5.9 \%$. Within low GC windows, $17 \%$ were without annotations (Figure 3A). Analysing the dinucleotide composition across the $100 \mathrm{bp}$ windows using the RIP composite index, showed that $33.1 \%$ of the genome have a composite index value above zero and are thereby interpreted as affected by RIP. Only $19.3 \%$ of the windows with GC content above $33.2 \%$ are 
RIP affected, whereas $95.7 \%$ of the low GC peak are affected by RIP (Figure 3B). Across GC levels, $82 \%$ of transposable elements are affected by RIP. Similarly, $83 \%$ of unknown repeats are RIP affected.

M. acridum ARSEF 324 is homozygous for the MAT1-2 mating-type ideomorph (Figure 4). The two mating-type alleles of $M$. acridum ARSEF 324 and the mating type allele of M. acridum CQMa 102 have notable differences in insertions. H1 contains a Mutator-like element (MULE) DNA transposon downstream of MAT1-2-3 not present in H2, contrary H2 harbours a LAGLIDAG homing endonuclease not present in H1. M. acridum CQMa 102 contains a WD-repeat domain that is not found in either of the two ARSEF 324 alleles.

\section{Genome comparison}

In order to assess if our haplotig assembly differed from other species within the genus Metarhizium, we compared the assembly of strain ARSEF 324 with genomes of from eight Metarhizium species (Figure 5). For the phylogenetic analysis, two strains of Pochonia chlamydosporia representing the closest known sister taxa to the Metarhizium genus was included. The Metarhizium genomes presented range in haploid size from $30.8 \mathrm{Mb}$ to $44.7 \mathrm{Mb}$. The 12.536 predicted genes within ARSEF $324(\mathrm{H} 1)$, fall within the range predicted for other Metarhizium isolates, i.e. 8472 to 13,646 . A notable difference can be seen in the proportion of repetitive elements between genomes. The median repeat level across the Metarhizium isolates is $6.1 \%, M$. anisopliae strain JEF-290 has twice this, and the diploid M. acridum strain ARSEF 324 has three times this level, with $20.8 \%$ of the genome comprised of repetitive elements. The proportion of the genome of $M$. acridum strain ARSEF 324 that is affected by RIP is likewise higher than what was observed for the other genomes analysed (Figure 5). A conservative estimate of the RIP affected region can be given by only including regions that are assessed as RIP affected with all three RIP calculating methods: RIP product, RIP substrate and RIP composite as used above. With this conservative estimate, $17.9 \%$ of the M. acridum strain ARSEF 324 genome is affected by RIP (calculated in $1000 \mathrm{bp}$ window, in $500 \mathrm{bp}$ steps). Evaluating the genomes ordered in decending order of RIP content, the diploid M. acridum strain is followed by M. acridum CQMa 102 and M. rileyi ARSEF 4871 with 3.8\% and 3.6\% respectively (Figure 5). The effect of window size on total RIP proportions is shown in Table S3.

\section{DISCUSSION}

Metarhizium species are predominately haploid, with only two taxa within the M. majus complex described as stable diploids. Here we present a highly improved genome assembly of $M$. acridum based on long-read sequencing data, which is assembled across repeat regions. This reveals a genome enriched in repetitive elements relative to other Metarhizium isolates or species studied. All presented analyses indicate that the sequences $M$. acridum strain ARSEF 324 show the genomic characteristics of a diploid. All 35 contigs of the representative assembled haplotype, were subject to the mapping of smaller contigs and all were heterozygous with allele frequencies between reads around 0.5 . The only exceptions are the two minor contigs 17 and contig 34, which could be examples of misassembly, or segmental aneuploidy.

A growing body of evidence indicates that CCNV is a common adaptive trait of many fungi and has been observed at low prevalence in natural populations. The effect on fitness induced by CCNVs has been shown to vary, and can be life-stage specific, and how often these variants are reproductively stable is unknown. We did not see any signs of chromosomal loss to indicate ongoing haploidisation 
within this diploid strain of $M$. acridum. The absence of chromosome loss suggests that the polyploidisation is recent, or that the strain constitutes a stable diploid. Kepler et al. (2016) found monophyletic clades of diploid isolates in a population genetic study of the $M$. majus species complex (the MGT clade, sensu Bischoff et al 2009). A similar population study could shed light on the prevalence of diploidy or CCNV within M. acridum. Alternatively, the diploid stability can be assessed by long-term evolution experiment focusing on possible reversions to the ancestral haploid state. Future research is needed to investigate gene evolution within this strain and identify concerted gene loss levels and rates of pseudo- and neofunctionalisation. If the diploidisation of this strain is not recent, it should be possible to trace divergence between ortholog genes (paralogs, if the diploidisation is the result of endoreplication), where gene duplication did not result in increased fitness and purifying selection.

The highly continuous genome assembly ARSEF 324 includes the highest proportion of repetitive elements of any Metharhizium species reported so far. Part of the reason for this is likely to do with the fragmented character of many short read based assemblies and the inherent bias that spring from difficulties in assembling across repeats using these technologies. Our comparative analysis includes, besides the genome assembly of $M$. acridum ARSEF 324, three assemblies based on long-read technologies, i.e. M. anisopliae JEF-290 M. brunneum ARSEF 4556 and P. chlamydosporia 170 (Table S2). Assuming that these assemblies give credible indications of their species' general repeat content, it is noticeable that two Metarhizium species, both belonging to the presumable asexual PARB clade and have considerable less repetitive elements compared with ARSEF 324. This difference between the primarily asexual $M$. anisopliae and $M$. brunneum and the sexual $M$. acridum corroborates the theory that sexual reproduction allows for the proliferation of transposable elements within populations like 'a sexually-transmitted nuclear parasite' (Hickey, 1982). Another difference between asexual and sexual fungal species are found in their defense against such selfish DNA elements. The RIP machinery that mutate TE's, is only active during the sexual cycle and should therefore only be observed in species that sexually recombine and to a less degree in primarily asexually reproducing species. Comparing the repeat content to the proportion of the genome affected by RIP across the Metarhizium species show that the two well-assebled genomes of $M$. anisopliae JEF-290 and $M$. brunneum ARSEF 4556 both have a higher ratio of 'repeats \%' to 'RIP \%' compared to M. acridum ARSEF 324, indicating a lack of an active RIP control of repeats. Both the high repeat content and the active RIP defence supports that $M$. acridum is sexually reproducing, even though no teleomorph is known. The RIP defence of $M$. acridum has shaped the genome by changing the nucleotide composition of approximately one-fifth of the genome, reducing the GC content in affected regions to less than half of the background GC frequency (51\%).

The presence of identical mating-type ideomorphs makes it unlikely that the diploidisation results from a mating event as hypothesised of the diploid strains within the M. majus species complex. This supports a model where either endoreplication, or allopolyploidsation through parasexuality are responsible for diploidisation. If the former were the case, the duplication event should be old enough for the measurable sequence divergence to have occurred between the homologous genes. The phylogenetic distances between homologous genes within the diploid were comparable to the distance observed between two $M$. robertsii isolates that were isolated with 27 years apart in the USA (Figure S3). This indicates that the observed phylogenetic distance between ARSEF 324 orthologs is consistent with the allopolyploidisation hypothesis. The high prevalence of parasexuality observed in $M$. robertsii renders alloploidisation a likely mode of forming the observed diploid M. acridum isolate. 
CCNV has thoroughly been linked with both stress and enhanced fitness. It is possible that the high frequency of parasexuality observed in coinfected insects (Riba et al., 1980; Leal-Bertioli et al., 2000; Wang et al., 2011) not just provides a means of recombination but also induce developmental changes that could be beneficial facing a host immune response. If the diploidisation of ARSEF 324 arose from a parasexual fusion, the question remains how stable or long-lived this is. The diploid strain ARSEF 324 is likely to be phenotypically different from haploid M. acridum strains. Higher ploidy can enable increased transcription of virulence factors or effectors, and it could be speculated that this could make the strain a more potent biocontrol agent. Diploidy can also work as a shield against detrimental mutation through functional redundancy (Haldane, 1932, p. 110; Orr, 1995) This could explain the strain specific high tolerance to UV-B radiation of ARSEF 324 compared to other $M$. acridum isolates reported by Braga et al. (2001).

While the diploidy reported here for an isolate within M. acridum is independent from the cases of diploidy in M. majus (Kepler et al., 2016), it is noteworthy that there are several instances of ploidy variation in the genus Metarhizium. It is tempting to speculate whether the complex species associations where many isolates are soil-dwelling plant-root endophytes and rhizosphere colonisers as well as potent insect pathogens influence the tendency for ploidy-level variation (St-Leger and Wang 2020). It certainly provides the potential for these fungi to find themselves in stressful microhabitats, and it has been noted that stress is the rule rather than the exemption for Metarhizium (Lovett and St. Leger, 2015).

\section{ACKNOWLEDGEMENTS}

HHDFL was supported by a Sapere Aude Grant from the Independent Research Fund Denmark. JES is a CIFAR Fellow in the program Fungal Kingdom: Threats and Opportunities and was supported by NSF DEB 1441715, NSF DEB 1557110, and USDA-NIFA Hatch project CA-R-PPA-5062-H.

\section{AUTHOR CONTRIBUTIONS}

HHDFL initiated the project. MN cultured fungi, performed DNA extraction and initial data QC. KNN performed genome assembly, JFMS and JS carried out genome annotation, KNN and JFMS carried out all other data analyses with input from HHDFL and JS, KNN drafted the manuscript and all authors were involved in the finalisation of the manuscript.

\section{DATA AVAILABILITY}

Data deposition: Sequencing data and genome assembly of Metarhizium acridum ARSEF 324 has been deposited and are available under the Umbrella Bioproject accession: (Under preparation)

Genbank accession numbers of all genomes used in this study are given in supplementary table S2. 


\section{LITERATURE CITED}

Aguileta, G. et al. (2014) 'High Variability of Mitochondrial Gene Order among Fungi', Genome Biology and Evolution, 6(2), pp. 451-465. doi: 10.1093/gbe/evu028.

Albertin, W. and Marullo, P. (2012) 'Polyploidy in fungi : evolution after whole-genome duplication', Preceedings of The Royal Society B, 279(April), pp. 2497-2509. doi: 10.1098/rspb.2012.0434.

Almagro Armenteros, J. J. et al. (2019) 'SignalP 5.0 improves signal peptide predictions using deep neural networks', Nature Biotechnology. Springer US, 37(4), pp. 420-423. doi: 10.1038/s41587-0190036-z.

Bao, W., Kojima, K. and Kohany, O. (2015) 'Repbase Update, a database of repetitive elements in eukaryotic genomes', Mobile DNA, 6(11). Available at: https://www.girinst.org/.

Bao, Z. and Eddy, S. R. (2002) 'Automated de novo identification of repeat sequence families in sequenced genomes', Genome Research. doi: 10.1101/gr.88502.

Barkla, B. J. et al. (2018) 'Making Epidermal Bladder Cells Bigger: Developmental- and Salinity-Induced Endopolyploidy in a Model Halophyte', Plant Physiology, 177(2), pp. 615-632. doi: 10.1104/pp.18.00033.

Bateman, A. et al. (2021) 'UniProt: the universal protein knowledgebase in 2021', Nucleic Acids Research, 49(D1), pp. D480-D489. doi: 10.1093/nar/gkaa1100.

Besemer, J. and Borodovsky, M. (2005) 'GeneMark: Web software for gene finding in prokaryotes, eukaryotes and viruses', Nucleic Acids Research, 33(SUPPL. 2), pp. 451-454. doi: 10.1093/nar/gki487.

Bischoff, J. F., Rehner, S. A. and Humber, R. A. (2009) 'A multilocus phylogeny of the Metarhizium anisopliae lineage', Mycologia, 101(4), pp. 512-530. doi: 10.3852/07-202.

Biskup, A. and Izmaiłow, R. (2004) 'Endosperm development in seeds of Echium vulgare L. (Boraginaceae) from polluted sites', Acta Biologica Cracoviensia Series Botanica.

Blin, K. et al. (2019) 'antiSMASH 5.0: updates to the secondary metabolite genome mining pipeline', Nucleic Acids Research, 47(W1), pp. W81-W87. doi: 10.1093/nar/gkz310.

De Bodt, S., Maere, S. and Van de Peer, Y. (2005) 'Genome duplication and the origin of angiosperms', Trends in Ecology \& Evolution, 20(11), pp. 591-597. doi: 10.1016/j.tree.2005.07.008.

Bolger, A. M., Lohse, M. and Usadel, B. (2014) 'Trimmomatic: A flexible trimmer for Illumina sequence data', Bioinformatics. doi: 10.1093/bioinformatics/btu170.

Braga, G. U. L. et al. (2001) 'Variability in Response to UV-B among Species and Strains of Metarhizium Isolated from Sites at Latitudes from $61^{\circ} \mathrm{N}$ to $54^{\circ} \mathrm{S}^{\prime}$, Journal of Invertebrate Pathology, 78(2), pp. 98108. doi: 10.1006/jipa.2001.5048.

Bridges, C. B. (1919) 'Duplication', Anat Rec, 15, pp. 357-358.

Cookson, S. J., Radziejwoski, A. and Granier, C. (2006) 'Cell and leaf size plasticity in Arabidopsis: what is the role of endoreduplication?', Plant, cell \& environment, 29(7), pp. 1273-83. doi: 10.1111/j.13653040.2006.01506.x.

Crow, K. D. and Wagner, G. P. (2006) 'What is the role of genome duplication in the evolution of complexity and diversity?', Molecular Biology and Evolution, 23(5), pp. 887-892. doi: 10.1093/molbev/msj083. 
Dietrich, F. S. (2004) 'The Ashbya gossypii Genome as a Tool for Mapping the Ancient Saccharomyces cerevisiae Genome', Science, 304(5668), pp. 304-307. doi: 10.1126/science.1095781.

Dupeyron, M. et al. (2019) 'Evolution of Mutator transposable elements across eukaryotic diversity', Mobile DNA. Mobile DNA, 10(1). doi: 10.1186/s13100-019-0153-8.

Eddy, S. R. (2011) 'Accelerated Profile HMM Searches', PLoS Computational Biology. Edited by W. R. Pearson, 7(10), p. e1002195. doi: 10.1371/journal.pcbi.1002195.

Ellinghaus, D., Kurtz, S. and Willhoeft, U. (2008) 'LTRharvest, an efficient and flexible software for de novo detection of LTR retrotransposons', BMC Bioinformatics, 9(1), p. 18. doi: 10.1186/1471-2105-918.

Emms, D. M. and Kelly, S. (2019) 'OrthoFinder: phylogenetic orthology inference for comparative genomics', Genome Biology, 20(1), p. 238. doi: 10.1186/s13059-019-1832-y.

Farrer, R. A. et al. (2013) 'Chromosomal copy number variation, selection and uneven rates of recombination reveal cryptic genome diversity linked to pathogenicity.', PLoS genetics. Edited by J. Heitman, 9(8), p. e1003703. doi: 10.1371/journal.pgen.1003703.

Fox, D. T. and Duronio, R. J. (2013) 'Endoreplication and polyploidy: insights into development and disease', Development, 140(1), pp. 3-12. doi: 10.1242/dev.080531.

Galagan, J. E. and Selker, E. U. (2004) 'RIP: the evolutionary cost of genome defense', Trends in Genetics, 20(9), pp. 417-423. doi: 10.1016/j.tig.2004.07.007.

Gao, Q. et al. (2011) 'Genome sequencing and comparative transcriptomics of the model entomopathogenic fungi Metarhizium anisopliae and M. acridum', PLoS Genetics, 7(1). doi: 10.1371/journal.pgen.1001264.

Gerstein, A. C. et al. (2006) 'Genomic Convergence toward Diploidy in Saccharomyces cerevisiae', PLoS Genetics, 2(9), pp. 2-7. doi: 10.1371/journal.pgen.0020145.

Glass, N. L. and Dementhon, K. (2006) 'Non-self recognition and programmed cell death in filamentous fungi', Current Opinion in Microbiology, 9(6), pp. 553-558. doi: 10.1016/j.mib.2006.09.001.

Grabherr, M. G. et al. (2011) 'Full-length transcriptome assembly from RNA-Seq data without a reference genome', Nature Biotechnology. doi: 10.1038/nbt.1883.

Guy, L., Roat Kultima, J. and Andersson, S. G. E. (2010) 'genoPlotR: comparative gene and genome visualization in R', Bioinformatics, 26(18), pp. 2334-2335. doi: 10.1093/bioinformatics/btq413.

Haas, B. J. et al. (2008) 'Automated eukaryotic gene structure annotation using EVidenceModeler and the Program to Assemble Spliced Alignments', Genome Biology, 9(1), pp. 1-22. doi: 10.1186/gb-20089-1-r7.

Haldane, J. B. S. (1932) The Causes of Evolution. London, New York and Toronto: Longmans, Green.

Hickey, D. A. (1982) 'Selfish DNA: A sexually transmitted nuclear parasite', Genetics, 101(3-4), pp. 519531.

Hoff, K. J. et al. (2016) 'BRAKER1: Unsupervised RNA-Seq-based genome annotation with GeneMarkET and AUGUSTUS', Bioinformatics, 32(5), pp. 767-769. doi: 10.1093/bioinformatics/btv661.

$\mathrm{Hu}, \mathrm{X}$. et al. (2014) 'Trajectory and genomic determinants of fungal-pathogen speciation and host adaptation', Proceedings of the National Academy of Sciences of the United States of America, 111(47), pp. 16796-16801. doi: 10.1073/pnas.1412662111.

Hubley, R. et al. (2016) 'The Dfam database of repetitive DNA families', 44(November 2015), pp. 81- 
89. doi: 10.1093/nar/gkv1272.

Huerta-Cepas, J. et al. (2017) 'Fast Genome-Wide Functional Annotation through Orthology Assignment by eggNOG-Mapper', Molecular Biology and Evolution, 34(8), pp. 2115-2122. doi: 10.1093/molbev/msx148.

Huerta-Cepas, J. et al. (2019) 'eggNOG 5.0: a hierarchical, functionally and phylogenetically annotated orthology resource based on 5090 organisms and 2502 viruses', Nucleic Acids Research, 47(D1), pp. D309-D314. doi: 10.1093/nar/gky1085.

Jones, P. et al. (2014) 'InterProScan 5: Genome-scale protein function classification', Bioinformatics, 30(9), pp. 1236-1240. doi: 10.1093/bioinformatics/btu031.

Käll, L., Krogh, A. and Sonnhammer, E. L. L. (2007) 'Advantages of combined transmembrane topology and signal peptide prediction-the Phobius web server', Nucleic Acids Research. doi: 10.1093/nar/gkm256.

Kellis, M., Birren, B. W. and Lander, E. S. (2004) 'Proof and evolutionary analysis of ancient genome duplication in the yeast Saccharomyces cerevisiae', Nature, 428(6983), pp. 617-624. doi: 10.1038/nature02424.

Kepler, R. M. et al. (2016) 'Independent origins of diploidy in Metarhizium', Mycologia, 108, pp. 10911103. doi: 10.3852/15-270.

Kim, D. et al. (2019) 'Graph-based genome alignment and genotyping with HISAT2 and HISATgenotype', Nature Biotechnology. doi: 10.1038/s41587-019-0201-4.

Kokot, M., Długosz, M. and Deorowicz, S. (2017) 'KMC 3: counting and manipulating k-mer statistics', Bioinformatics. Edited by B. Berger, 33(17), pp. 2759-2761. doi: 10.1093/bioinformatics/btx304.

Koren, S. et al. (2017) 'Canu: Scalable and accurate long-read assembly via adaptive k-mer weighting and repeat separation', Genome Research, 27(5), pp. 722-736. doi: 10.1101/gr.215087.116.

Kronstad, J. W. et al. (2011) 'Expanding fungal pathogenesis: Cryptococcus breaks out of the opportunistic box', Nature Reviews Microbiology. Nature Publishing Group, 9(3), pp. 193-203. doi: 10.1038/nrmicro2522.

Leal-Bertioli, S. C. M. et al. (2000) 'Genetic exchange in Metarhizium anisopliae strains coinfecting Phaedon cochleariae is revealed by molecular markers', Mycological Research, 104(4), pp. 409-414. doi: 10.1017/S0953756299001549.

St. Leger, R. J. and Wang, J. B. (2020) 'Metarhizium : jack of all trades, master of many', Open Biology, 10(12), p. 200307. doi: 10.1098/rsob.200307.

Levasseur, A. and Pontarotti, P. (2011) 'The role of duplications in the evolution of genomes highlights the need for evolutionary-based approaches in comparative genomics', Biology Direct, 6(1), p. 11. doi: 10.1186/1745-6150-6-11.

Li, H. et al. (2009) 'The Sequence Alignment/Map format and SAMtools', Bioinformatics, 25(16), pp. 2078-2079. doi: 10.1093/bioinformatics/btp352.

Li, H. (2011a) 'A statistical framework for SNP calling, mutation discovery, association mapping and population genetical parameter estimation from sequencing data', Bioinformatics. doi: 10.1093/bioinformatics/btr509.

Li, H. (2011b) 'Improving SNP discovery by base alignment quality', Bioinformatics, 27(8), pp. 11571158. doi: 10.1093/bioinformatics/btr076. 
$\mathrm{Li}, \mathrm{H}$. (2013) Aligning sequence reads, clone sequences and assembly contigs with BWA-MEM. Available at: http://arxiv.org/abs/1303.3997.

Li, H. (2018) 'Minimap2: pairwise alignment for nucleotide sequences', Bioinformatics. Edited by I. Birol, 34(18), pp. 3094-3100. doi: 10.1093/bioinformatics/bty191.

Lisch, D. (2015) 'Mutator and MULE Transposons.', Microbiology spectrum. New York, NY: Springer New York, 3(2), pp. MDNA3-0032-2014. doi: 10.1128/microbiolspec.MDNA3-0032-2014.

Lombard, V. et al. (2014) 'The carbohydrate-active enzymes database (CAZy) in 2013', Nucleic Acids Research, 42(D1), pp. 490-495. doi: 10.1093/nar/gkt1178.

Lovett, B. and St. Leger, R. J. (2015) 'Stress is the rule rather than the exception for Metarhizium', Current Genetics. Springer Berlin Heidelberg, 61(3), pp. 253-261. doi: 10.1007/s00294-014-0447-9.

Lowe, T. M. and Eddy, S. R. (1997) 'tRNAscan-SE: A Program for Improved Detection of Transfer RNA Genes in Genomic Sequence', Nucleic Acids Research, 25(5), pp. 955-964. doi: 10.1093/nar/25.5.955.

Lynch, M. (2000) 'The Evolutionary Fate and Consequences of Duplicate Genes', Science, 290(5494), pp. 1151-1155. doi: 10.1126/science.290.5494.1151.

Marcet-houben, M. and Gabaldón, T. (2015) 'Beyond the Whole-Genome Duplication : Phylogenetic Evidence for an Ancient Interspecies Hybridization in the Baker's Yeast Lineage', pp. 1-26. doi: 10.1371/journal.pbio.1002220.

Mayrose, I. and Lysak, M. A. (2020) 'The Evolution of Chromosome Numbers: Mechanistic Models and Experimental Approaches', Genome Biology and Evolution. Edited by Y. Van De Peer, 13(2), pp. 1-15. doi: 10.1093/gbe/evaa220.

Metz, C. W. (1947) 'Duplication of Chromosome Parts as a Factor in Evolution', The American Naturalist, 81(797), pp. 81-103. Available at: https://www.jstor.org/stable/2457862.

Monjardino, P., Smith, A. G. and Jones, R. J. (2006) 'Zein transcription and endoreduplication in maize endosperm are differentially affected by heat stress', Crop Science, 46(6), pp. 2581-2589. doi: 10.2135/cropsci2006.03.0136.

Moore, D., Robson, G. D. and Trinci, A. P. J. (2011) 21st Century Guidebook to Fungi. Edited by A. P. J. Trinci. Cambridge University Press.

Ohno, S. (1970) Evolution by Gene Duplication, Evolution by Gene Duplication. Berlin, Heidelberg: Springer Berlin Heidelberg. doi: 10.1007/978-3-642-86659-3.

Okagaki, L. H. et al. (2010) 'Cryptococcal Cell Morphology Affects Host Cell Interactions and Pathogenicity', PLoS Pathogens. Edited by A. P. Mitchell, 6(6), p. e1000953. doi: 10.1371/journal.ppat.1000953.

Orr, H. A. (1995) 'Somatic mutation favors the evolution of diploidy', Genetics, 139(3), pp. 1441-1447. doi: 10.1093/genetics/139.3.1441.

Peter, J. et al. (2018) 'Genome evolution across 1,011 Saccharomyces cerevisiae isolates', Nature, 556(7701), pp. 339-344. doi: 10.1038/s41586-018-0030-5.

Price, A. L., Jones, N. C. and Pevzner, P. A. (2005) 'De novo identification of repeat families in large genomes', Bioinformatics, 21(Suppl 1), pp. i351-i358. doi: 10.1093/bioinformatics/bti1018.

Ranallo-Benavidez, T. R., Jaron, K. S. and Schatz, M. C. (2020) 'GenomeScope 2.0 and Smudgeplot for reference-free profiling of polyploid genomes', Nature Communications, 11(1), p. 1432. doi: 10.1038/s41467-020-14998-3. 
Rawlings, N. D., Barrett, A. J. and Finn, R. (2016) 'Twenty years of the MEROPS database of proteolytic enzymes, their substrates and inhibitors', Nucleic Acids Research, 44(D1), pp. D343-D350. doi: 10.1093/nar/gkv1118.

Riba, G. et al. (1980) 'Isolement de recombines mitotiques stables de type intermediaire chez Metarhizium anisopliae (Metschnikoff) par hybridation de biotypes sauvages', Comptes Rendus des Seances de I'Academie de Sciences de Paris D, pp. 657-660.

Roelofs, D. et al. (2020) 'Multi-faceted analysis provides little evidence for recurrent whole-genome duplications during hexapod evolution', BMC Biology. BMC Biology, 18(1), p. 57. doi: 10.1186/s12915020-00789-1.

Rosenblum, E. B. et al. (2013) 'Complex history of the amphibian-killing chytrid fungus revealed with genome resequencing data', Proceedings of the National Academy of Sciences, 110(23), pp. 93859390. doi: 10.1073/pnas.1300130110.

Sacerdot, C. et al. (2018) 'Chromosome evolution at the origin of the ancestral vertebrate genome', Genome Biology. Genome Biology, 19(1), p. 166. doi: 10.1186/s13059-018-1559-1.

Schneider, C. A., Rasband, W. S. and Eliceiri, K. W. (2012) 'NIH Image to ImageJ: 25 years of image analysis', Nature Methods. Nature Publishing Group, 9(7), pp. 671-675. doi: 10.1038/nmeth.2089.

Scholes, D. R. and Paige, K. N. (2015) 'Plasticity in ploidy: a generalized response to stress', Trends in Plant Science. Elsevier Ltd, 20(3), pp. 165-175. doi: 10.1016/j.tplants.2014.11.007.

Sher, N. et al. (2013) 'Fundamental differences in endoreplication in mammals and Drosophila revealed by analysis of endocycling and endomitotic cells', Proceedings of the National Academy of Sciences, 110(23), pp. 9368-9373. doi: 10.1073/pnas.1304889110.

Simão, F. A. et al. (2015) 'BUSCO: Assessing genome assembly and annotation completeness with single-copy orthologs', Bioinformatics, 31(19), pp. 3210-3212. doi: 10.1093/bioinformatics/btv351.

Sionov, E. et al. (2010) 'Cryptococcus neoformans Overcomes Stress of Azole Drugs by Formation of Disomy in Specific Multiple Chromosomes', PLoS Pathogens. Edited by S. G. Filler, 6(4), p. e1000848. doi: 10.1371/journal.ppat.1000848.

Slater, G. S. C. and Birney, E. (2005) 'Automated generation of heuristics for biological sequence comparison', BMC Bioinformatics, 6, pp. 1-11. doi: 10.1186/1471-2105-6-31.

Smit, A., Hubley, R. and Green, P. (2015) 'RepeatMasker'. Open-4.0. Available at: http://www.repeatmasker.org.

Stanke, M. and Morgenstern, B. (2005) 'AUGUSTUS : a web server for gene prediction in eukaryotes that allows user-defined constraints', 33, pp. 465-467. doi: 10.1093/nar/gki458.

Wang, C. and St. Leger, R. J. (2005) 'Developmental and Transcriptional Responses to Host and Nonhost Cuticles by the Specific Locust Pathogen Metarhizium anisopliae var. acridum', Eukaryotic Cell, 4(5), pp. 937-947. doi: 10.1128/EC.4.5.937-947.2005.

Wang, S. et al. (2011) 'Local adaptation of an introduced transgenic insect fungal pathogen due to new beneficial mutations', Proceedings of the National Academy of Sciences, 108(51), pp. 20449-20454. doi: $10.1073 /$ pnas.1113824108.

Wolfe, K. H. and Shields, D. C. (1997) 'Molecular evidence for an ancient duplication of the entire yeast genome', Nature. doi: 10.1038/42711.

Zaragoza, O. et al. (2010) 'Fungal Cell Gigantism during Mammalian Infection', PLoS Pathogens. Edited by A. P. Mitchell, 6(6), p. e1000945. doi: 10.1371/journal.ppat.1000945. 
bioRxiv preprint doi: https://doi.org/10.1101/2021.03.15.435421; this version posted March 16, 2021. The copyright holder for this preprint (which was not certified by peer review) is the author/funder, who has granted bioRxiv a license to display the preprint in perpetuity. It is made available under aCC-BY-NC-ND 4.0 International license.

Zhang, S., Ren, L. Y. and Zhang, Y. J. (2020) 'Complete mitogenome of the entomopathogenic fungus Metarhizium rileyi', Mitochondrial DNA Part B: Resources. doi: 10.1080/23802359.2020.1742596. 


\section{TABLES AND FIGURES}

\section{A) GenomeScope Profile}

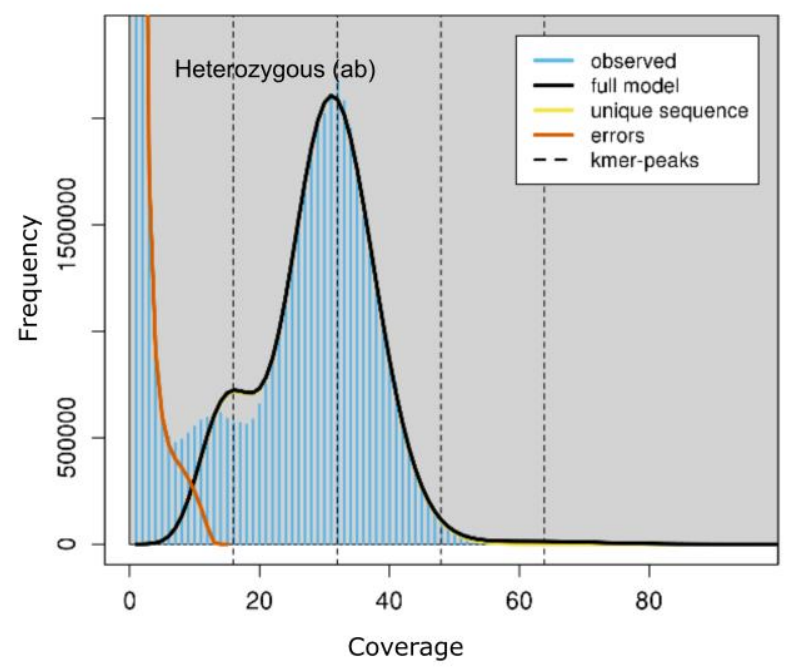

B) SmudgePlot

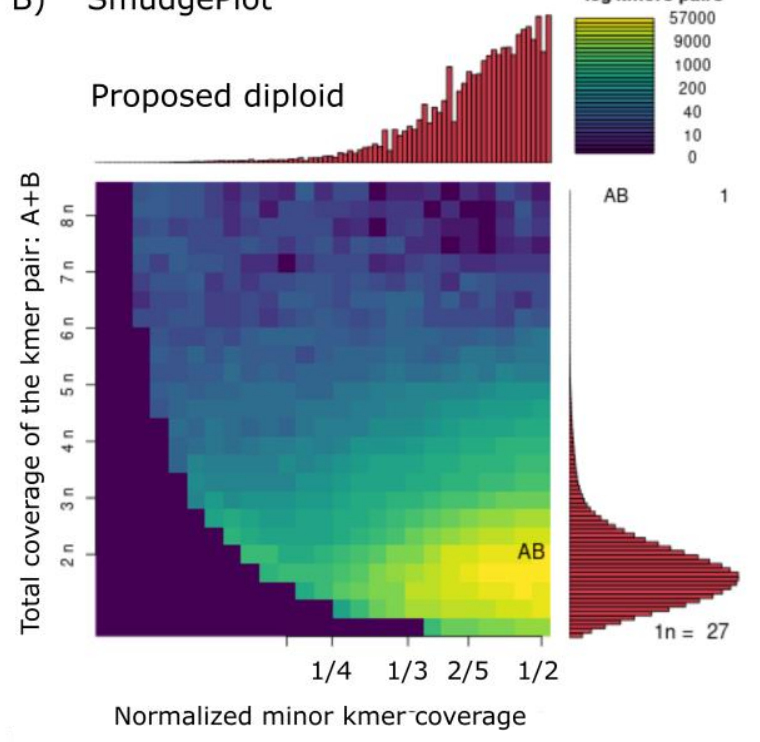

Fig. 1

The kmer analysis for Metarhizium acridum ARSEF 324 PacBio subread data revealed a diploid structure of heterozygous kmer pairs. A) Based on the the number of unique 21-mers the genome is estimated to have a haploid length of $45 \mathrm{Mb}$, with $18.2 \%$ repeats. The two peaks of the k-mer frequency profile of the observed data, where the lesser have half the read coverage of the higher peak, indicate heterozygocity, consistent with diploidy. The low frequency of k-mers from heterozygous loci indicate that most 21 -mer were heterozygous $(0.5 \%)$. B) The diploidy of the genome was confermed by Smudgeplot analysis, comparing the sum of kmer pair coverages (CovA + CovB) to their relative coverage (CovB / (CovA + CovB)). No higher level ploidy was observed. Max and min estimates from the GenomeScope analysis is available in supplementary material table 1. 
bioRxiv preprint doi: https://doi.org/10.1101/2021.03.15.435421; this version posted March 16, 2021. The copyright holder for this preprint (which was not certified by peer review) is the author/funder, who has granted bioRxiv a license to display the preprint in perpetuity. It is made available under aCC-BY-NC-ND 4.0 International license.

$H 1$, representative assembly of the haploid genome $\mathrm{H} 2$, contigs mapped to $\mathrm{H} 1$

Depth of reads mapped to $\mathrm{H} 1$ :
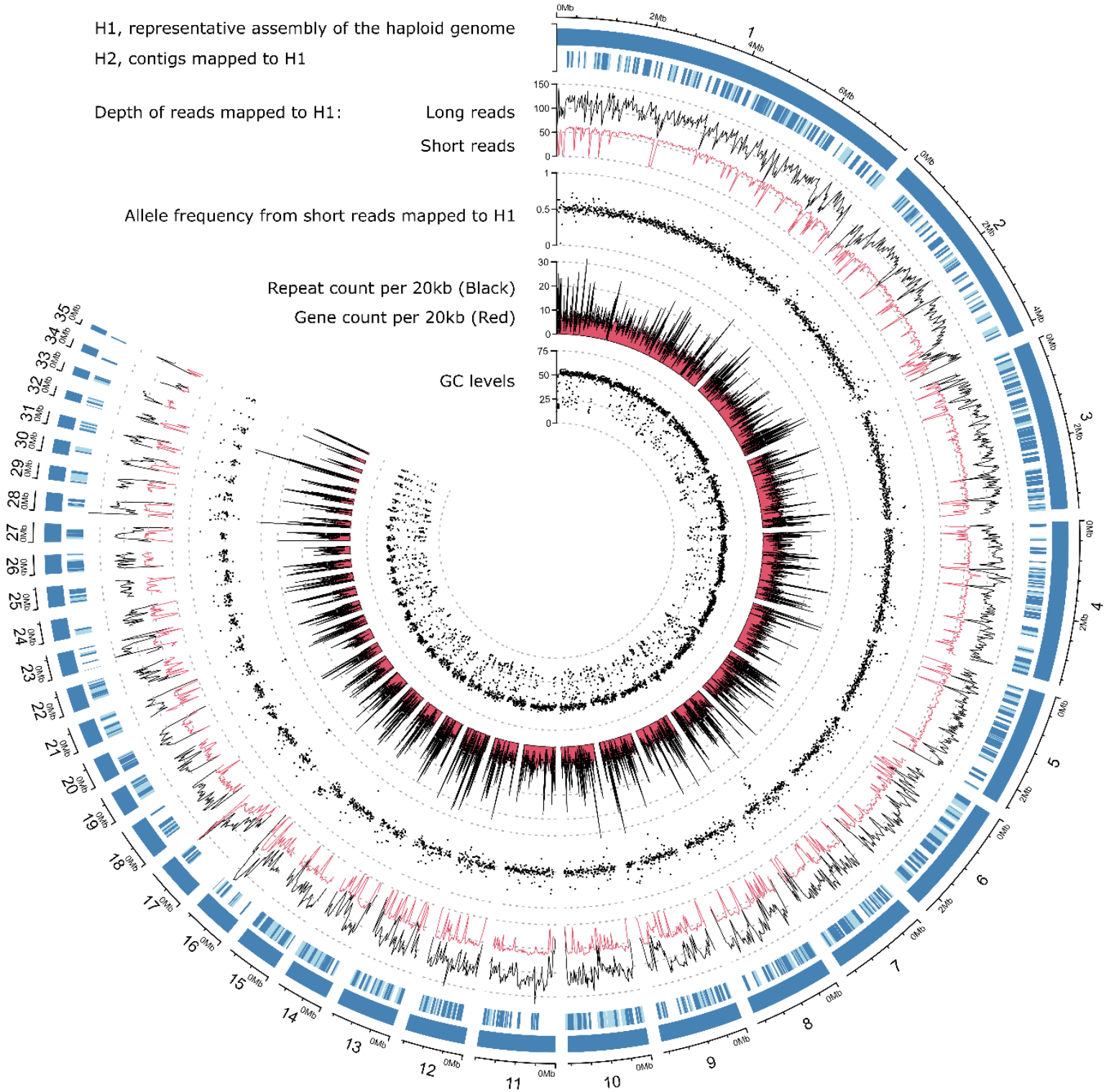

Fig. 2

Genome-wide statistics of sequencing data, assembly and traits of the Metarhizium acridum strain ARSEF 324 genome. The plot consists of five major tracks; from the outside: i) 549 contigs, 35 contigs placed in the outer band $(\mathrm{H} 1)$ represent the haploid genome. The remaining contigs $(\mathrm{H} 2)$ map to $\mathrm{H} 1$. $\mathrm{H} 2$ contigs are given in light and dark blue, where dark blue indicates the fraction of each contig mapped to $\mathrm{H} 1$. The following tracks, show ii) the read depth of long reads (PacBio) and short reads (BGI DBNseq) mapped to H1 (mean depth within $20 \mathrm{~kb}$ windows), iii) Allele frequencies calculated from short reads (BGI DBNseq) mapped to $\mathrm{H} 1$ (mean within $10 \mathrm{~kb}$ windows), iv) Repeat and gene count within $20 \mathrm{~kb}$ windows shown in black and red respectively, v) The GC levels within $10 \mathrm{~kb}$ windows. 

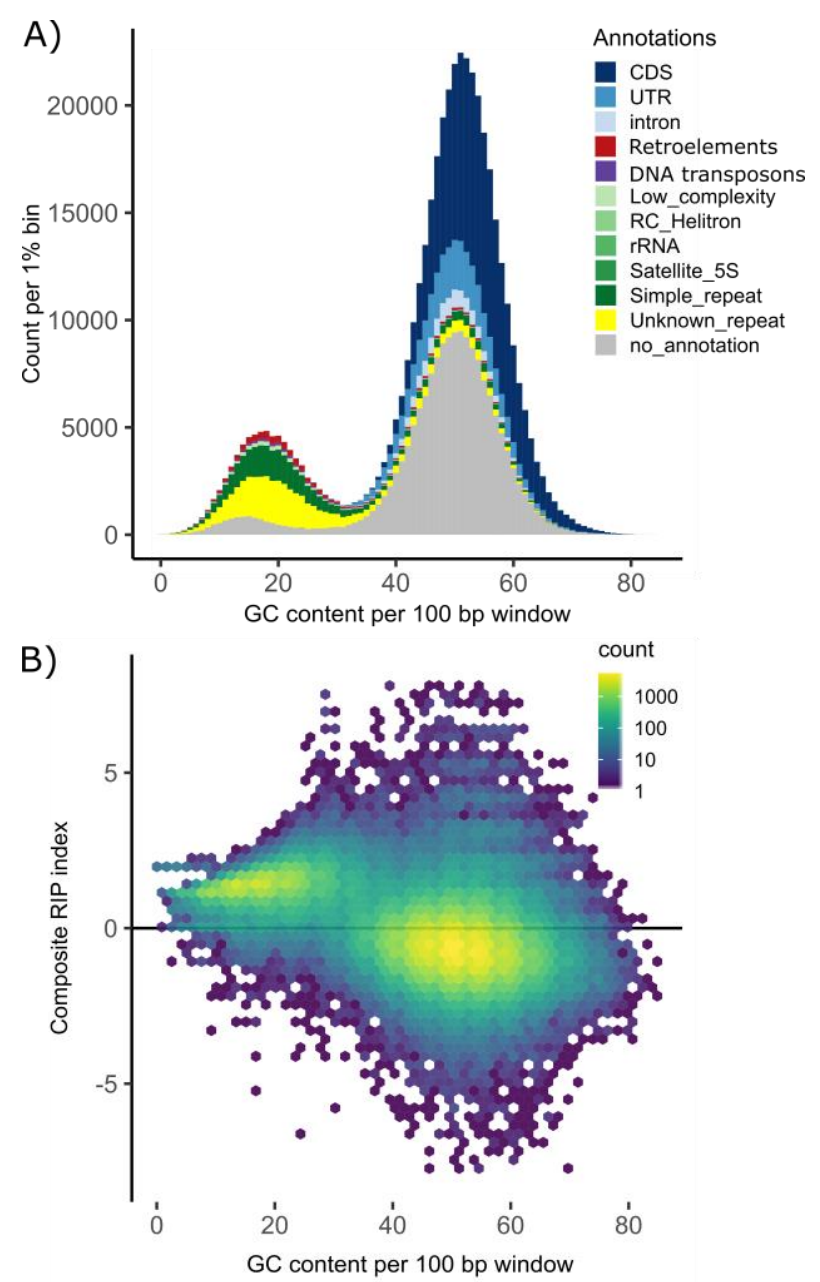

Fig. 3

GC content calculated in $100 \mathrm{bp}$. windows across the $44.7 \mathrm{Mb} \mathrm{H} 1$ haploid genome. A) If a window overlapped with more than half of its length with an annotated feature this were signed to the window. The bi-modial distribution have peaks GC-levels of $17.6 \%$ and $51.3 \%$. Each peak approximates normal distributions with standard deviations of 5.6 and 6.5, respectively. The fitted normal distributions intersect at $33.2 \%$; this level is set to split high and low GC values. Genomic windows with high GC values is largely comprised of CDS (37.3\%), UTR (10.9\%), introns (3.3\%) and sequence that were without annotations (42.3\%). Genomic windows with low GC values is dominated by repetitive elements. Of these repetitive elements $40.5 \%$ are unknown, $28.7 \%$ are simple repeats, $6.4 \%$ are retroelements, $5.9 \%$ are DNA transposons. Of the low GC windows $17 \%$ were without annotations. B) Composite RIP index as a function of GC content. Composite RIP index $>0$ are considered RIP affected, equaling $33.1 \%$ of the total genome, or $95.7 \%$ of the windows within the low GC peak. Combining different indices of RIP will provide a more conservative estimate of the proportion of the genome that is affected by RIP (17.9\%, Figure 5). 
bioRxiv preprint doi: $\mathrm{https}$ //doi.org/10.1101/2021.03.15.435421: this version posted March 16, 2021. The copyright holder for this preprint (which was not certified by peer review) is the author/funder, who has granted bioRxiv a license to display the preprint in perpetuity. It is made available under aCC-BY-NC-ND 4.0 International license.

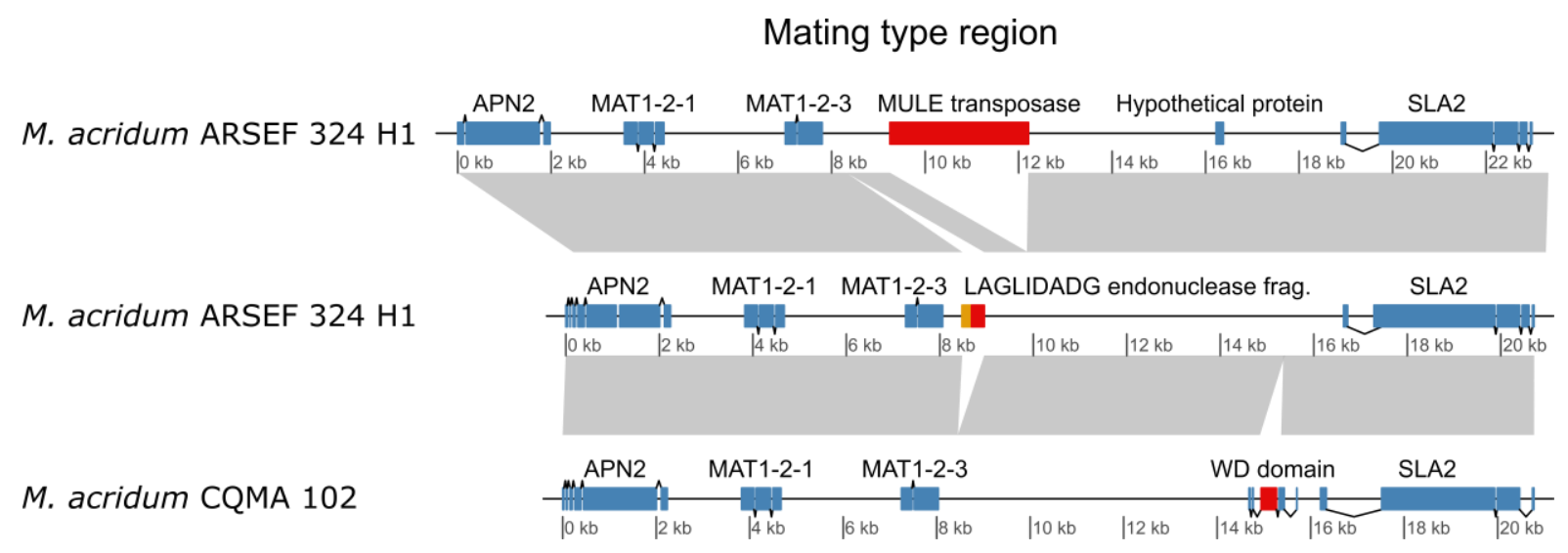

Fig. 4

Synteny between mating-type loci of the two alleles Metarhizium acridum strain ARSEF 324 and M. arcridum strain CQMa 102. The M. acridum strain ARSEF 324 is homozygous for the MAT1-2 mating-type ideomorph. Down-stream of the ARSEF H1 MAT1-2-3 gene, a 3 kb DNA transposon of the 'MULE' (Mutator-like elements) superfamily is found, this is not present in ARSEF H2. Mutator transposable elements are among the most mutagenic transposons known (Lisch, 2015; Dupeyron et al., 2019). Conversely, H2 harbours a LAGLIDADG endonuclease fragment (red) together with a fragment of the mitochondrial COX3 gene (orange), not present in $\mathrm{H} 1$. Neither of these insertions is present in the CQMa 102 strain, which contains a WD domain not present in ARSEF 324. 
bioRxiv preprint doi: https://doi.org/10.1101/2021.03.15.435421; this version posted March 16, 2021. The copyright holder for this preprint (which was not certified by peer review) is the author/funder, who has granted bioRxiv a license to display the preprint in perpetuity. It is made available under aCC-BY-NC-ND 4.0 International license.

\begin{tabular}{|c|c|c|c|c|c|c|c|c|}
\hline & Species & Strain & $\begin{array}{l}\text { Genome } \\
\text { size (Mb) }\end{array}$ & Genes & $\begin{array}{l}\text { Repetitive } \\
\text { elements (\%) }\end{array}$ & $\begin{array}{l}\text { No. of } \\
\text { contigs }\end{array}$ & L50 & $\begin{array}{l}\text { RIP affected } \\
(\%)\end{array}$ \\
\hline Pathogen to: & M. anisopliae & JEF 290 & 2.8 & 1364 & 12.64 & - 18 & - & 1.37 \\
\hline [ Insect (generalist) & M. brunneum & ARSEF 3297 & 37.1 & 1068 & 4.19 & 180 & - & 0.78 \\
\hline - Insect (Specialist) & M. brunneum & ARSEF 4556 & 37.8 & 1142 & 6.15 & - 8 & - & 2.76 \\
\hline Nematodes & M. anisopliae & ARSEF 549 & 38.5 & 1089 & 5.04 & 213 & - 14 & 2.16 \\
\hline & D. robertsii & ARSEF 23 & 41.7 & 1168 & 3.97 & 941 & - 41 & .0 .26 \\
\hline & M. robertsif & ARSEF 2575 & 40.3 & 1238 & 6.01 & 464 & 32 & 2.19 \\
\hline & M. guizhouense & ARSEF 977 & 43.5 & 1178 & 7.55 & 1111 & - 32 & 2.85 \\
\hline & M. majus & ARSEF 297 & 42.1 & 1153 & 5.47 & 1847 & 105 & -1.29 \\
\hline & - M. acridum & ARSEF $324 \mathrm{H} 1$ & 44.7 & 1253 & 20.80 & 35 & & 17.9 \\
\hline & M. acridum & ARSEF $324 \mathrm{H} 2$ & 39.2 & 1019 & & & & \\
\hline & M. acridum & CQMa 102 & 39.4 & 984 & 6.83 & 1608 & 211 & 3.77 \\
\hline & M. album & ARSEF 1941 & 30.5 & 847 & 6.74 & 731 & 36 & 3.4 \\
\hline & M. rileyi & ARSEF 4871 & 32 & 876 & 8.39 & 1000 & 32 & 3.6 \\
\hline & P. chlamydosporia & strain 123 & & 196 & 1.86 & 9087 & 850 & $\cdot 0.2$ \\
\hline & P. chlamydosporia & strain 170 & & 420 & 4.03 & 114 & 8 & - 1.39 \\
\hline
\end{tabular}

Fig. 5

A consensus maximum-likelihood phylogeny based on 444 known single-copy orthologous genes for sequenced Metarhizium species and the related Pochonia chlamydosporia. All splits within the phylogeny had $100 \%$ bootstrap support, except the artificial split between $\mathrm{H} 1$ and $\mathrm{H} 2$. From the left: ML phylogeny, tip icons indicating the host adaptation, species names and strain id. Genome size and number of genes is retrieved from NCBI Genbank and the protein file available under the accession found in table S2. The proportion of repetitive elements within each isolate was found as described in the method section. The figure highlights that the genome assembly of $M$. acridum ARSEF 324 generated in this study is the longest genome assembly of any Metarhizium isolate, and the one with the highest proportion of repetitive elements. 
bioRxiv preprint doi: https://doi.org/10.1101/2021.03.15.435421; this version posted March 16, 2021. The copyright holder for this preprint (which was not certified by peer review) is the author/funder, who has granted bioRxiv a license to display the preprint in perpetuity. It is made available under aCC-BY-NC-ND 4.0 International license.

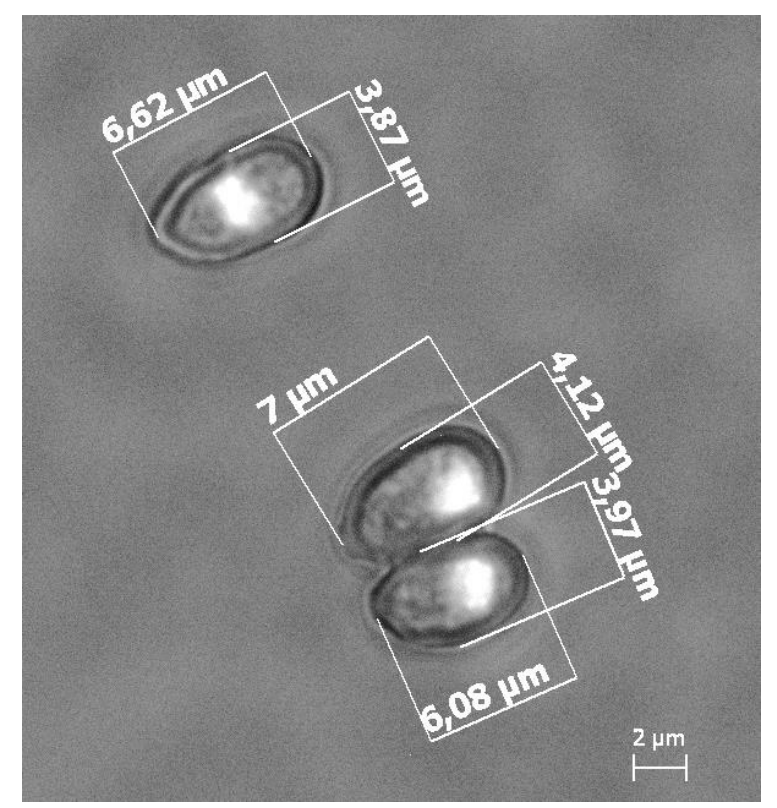

Fig. 6

DAPI stained nuclei in Metarhizium acridum strain ARSEF 324 conidia. All inspected spores contained monokaryons. 